\title{
The microbiology of head and neck space infections at the Maxillofacial Clinic at Livingstone Hospital
}

SADJ May 2020, Vol. 75 No. 4 p192 - p197

\author{
N Singh', S Ngwenya², J Molepo ${ }^{3}$
}

\begin{abstract}
Introduction

Head and neck space infections remain one of the most commonly encountered conditions at Maxillofacial clinics countrywide. Patients admitted with these infections tend to have prolonged hospital stays and often require intensive care support. This places financial and logistic constraints on our health care system. There are also growing concerns, worldwide, regarding antibiotic resistance.
\end{abstract}

\section{Aims and objectives}

To determine the microbial spectrum of head and neck space infections in patients admitted to the Maxillo-Facial clinic at Livingstone Hospital in the Eastern Cape over a period of 5 years.

\section{Methods}

Demographic, clinical and laboratory data was retrieved from the medical records of 140 patients presenting with head and neck space infections.

\section{Results}

Most patients were males aged 21-40 years. The most common cause of head and neck infections was nonodontogenic. The submandibular space was the most commonly implicated. Gram positive facultative anaerobes were most commonly identified.

\section{Author affiliations:}

1. Nerisha Singh: $B C h D, P D D$ (Implantology), MSc (Dentistry), MSc Dent Student, Department of Oral Pathology, School of Oral Health Sciences, Faculty of Health Sciences, University of the Witwatersrand, Johannesburg, South Africa.

ORCID Number: 0000-0002-2704-7031

2. Sizakele Ngwenya: BSC, BDS, MDent (Oral Pathology), Head, Department of Oral Pathology, School of Oral Health Sciences, Faculty of Health Sciences, University of the Witwatersrand, Johannesburg, South Africa.

ORCID Number: 0000-0002-9397-5178

3. Julitha Molepo: ND, BSc, BSc (Hons), MS, PhD, Head, Department of Oral Biological Sciences, School of Oral Health Sciences, Faculty of Health Sciences, University of the Witwatersrand, Johannesburg, South Africa. ORCID Number: 0000-0002-5547-097X

Corresponding author: Julitha Molepo

Head: Department of Oral Biological Sciences,

University of the Witwatersrand, Johannesburg,

Faculty of Health Sciences, School of Oral Health Sciences,

7 York Road, Parktown, South Africa.

Email: julitha.molepo@wits.ac.za

Author contributions:

1. Nerisha Singh: Conceptualisation, data collection, analysis and interpretation; first draft, revision, final write-up - 30\%

2. Sizakele Ngwenya: Conceptualisation and design, data analysis and interpretation, first draft, revision, final write-up and approval $-35 \%$

3. Julitha Molepo: Conceptualisation and design, data analysis and interpretation, first draft, revision, final write-up and approval - $35 \%$
Bacteroides species and Staphylococcus aureus displayed sensitivity to clindamycin and amoxicillin with clavulanic acid. Viridans streptococci were sensitive to both penicillin and clindamycin.

\section{Conclusion}

Bacteroides species were the most commonly isolated bacteria, followed by Viridans streptococci and Staphylococcus aureus.

\section{INTRODUCTION}

Patients admitted with deep space head and neck infections tend to have prolonged hospital stays and often require intensive care support, which places financial and logistic constraints on our health care system. There are also growing concerns, worldwide, regarding antibiotic resistance.

Several studies reported that odontogenic infections were identified as the most common source of head and neck infections. ${ }^{1-3}$ Infiltration through fascial spaces is an important factor to consider in the evaluation of head and neck space infections. The submandibular space was the most frequently involved fascial space in both single $e^{4-8}$ and multiple space infections. ${ }^{2,3}$

Head and neck space infections are caused by both aerobic and anaerobic microorganisms. The most frequently isolated aerobic bacterium was Streptococcus viridans $^{6}$ while Bacteroides was the most common anaerobic bacterium..$^{3,8}$ Molomo et al. ${ }^{5}$ and Cabral et al. ${ }^{9}$ reported Staphylococcus aureus to be the most commonly isolated organism.

Effective management consists of surgical drainage and the administration of the appropriate antibiotic. Staphylococcus aureus, Streptococcus mitis/oralis and Morganella morganii displayed high resistance levels against amoxicillin..$^{7,10}$

Amoxicillin with clavulanic acid was reported as the most effective antibiotic for the most commonly isolated organisms, ${ }^{7}$ therefore it was recommended as the empirical drug of choice in head and neck space infections. ${ }^{7,8}$

This study focussed on the microbial spectrum and antibiotic sensitivity patterns in patients with head and neck space infections over a 5 year period, in order to 
improve guidelines for appropriate antibiotic prescription, thus decreasing the length of hospital stays and contributing to the fight against antimicrobial resistance.

\section{MATERIALS AND METHODS}

\section{Study design}

This was a retrospective study which comprised the analysis of patient variables (age, gender and source of infection), microbial spectrum and antibiotic sensitivity patterns in patients presenting with head and neck space infections at the Maxillo-Facial Clinic, Livingstone Hospital, Eastern Cape, South Africa.

\section{Sampling method}

Cases were selected using the convenience sampling method. The admissions book at the Maxillofacial clinic was analysed to identify all patients with head and neck space infections admitted to the clinic at Livingstone Hospital from the $1^{\text {st }}$ of January 2012 to the $31^{\text {st }}$ of December 2016.

The medical records of all identified patients were reviewed. All patients who had microbial culture and sensitivity tests performed were included in the study. This study was approved by the Wits Human Research Ethics Committee (Ethical Clearance number: M170719), the Eastern Cape Department of Health and the National Health Laboratory Services.

\section{Data collection}

Demographic, clinical and laboratory data was retrieved from the medical records of 140 patients presenting with head and neck space infection. The variables analysed included age, gender, source of infection, fascial spaces involved, microorganisms identified and antibiotic sensitivity.

Microorganisms are considered to be sensitive when they cannot grow in the presence of a drug, resistant when they can grow and intermediate when a higher dose of the antibiotic is required to prevent growth.

\section{Data analysis}

Descriptive and inferential statistics were computed for all variables. Bivariate analysis was used for identifying associations. P-value based on the Chi-square test was utilised. A $p$-value of $<0.05$ was considered to be statistically significant.

\section{RESULTS}

\section{Patient demographic and clinical data}

The majority of patients presenting with head and neck space infections were male ( $n=97 ; 67 \%)$, while only 48 (33\%) were female. Eighty five patients (59\%) were 21 to 40 years of age. The youngest patient was 13 months and the oldest, 92 years old. Thirty six patients (25\%) fell into the 41-60 year age category, while 21 patients (14\%) were between 0-20 years old.
Head and neck infections appeared to be least common in patients older than 60 years, with only 3 patients $(2 \%)$ falling into this category.

Sixty four patients presented with an odontogenic cause of infection, while 81 presented with a nonodontogenic cause. Periapical lesions presented in the majority of patients with an odontogenic cause of infection ( $n=32 ; 50 \%)$, followed by alveolar osteitis $(n=22 ; 34 \%)$, pericoronitis $(n=4 ; 10 \%)$ and periodontitis $(n=4 ; 6 \%)$. Where the aetiology was non-odontogenic, 60 patients $(74 \%)$ presented with infection of an unknown aetiology, followed by trauma $(n=18 ; 22 \%)$.

\section{Distribution of fascial space involvement}

The majority $(n=81)$ of patients had only a single space involved, while 54 had multiple fascial space involvement. In 10 cases the fascial space remained unidentified. The submandibular space was the most commonly involved with a total of 50 (62\%) patients presenting with infection in this space.

Ten cases involved the buccal space (1\%), eight, the submental (10\%), six, the peri-orbital (8\%) and three, the zygomatic space (4\%). The submasseteric, parapharyngeal, superior labial and temporal spaces were less commonly affected and presented with one case each. These results are presented in Figure 1.

Of the 54 patients that presented with multiple space involvement, 31 (57\%) had two spaces affected, 22 (41\%) had three spaces affected and only one (2\%) had 4 fascial spaces being affected.

The most commonly implicated spaces in cases with multiple space involvement was the submandibular (35 cases, 64.9\%), followed by the sublingual (15 cases, $27.7 \%$ ) and submental (14 cases, 26\%) spaces (Figure 2).

\section{The spectrum of microorganisms isolated}

Thirty eight different types of microorganisms were isolated (Table 1). The most commonly isolated bacteria were Bacteroides species (16.7\%) followed by Viridans streptococci (11\%) and Staphylococcus aureus (8.6\%).

Less commonly isolated were coagulase negative Streptococcus (3.8\%), Streptococcus constellatus (4.8\%); alpha, beta and non-haemolytic Streptococcus (3.3\%); Morganella morganii species (3.8\%); Streptococcus anginosus (3.8\%) and Streptococcus mitis/oralis (3.8\%).

\section{Antibiotic sensitivities}

Bacteroides species, found in 35 specimens, was 100\% sensitive to metronidazole, carbapenems, piperacillin, clindamycin, cefoxitin, chloramphenicol and amoxicillin with clavulanic acid.

Six of the 25 isolates of Viridans streptococci showed resistance to erythromycin/azithromycin (24\%), while 15 displayed sensitivity (60\%). Four (16\%) of the isolates displayed resistance to clindamycin while 16 (64\%) showed sensitivity. 
Seven isolates showed sensitivity to penicillin and ampicillin (28\%) while only one showed resistance (4\%). Four isolates were sensitive to cefotaxime and ceftriaxone (16\%) while one (4\%) was resistant. Four isolates were sensitive to vancomycin (16\%) and one to linezolid (4\%).

Staphylococcus aureus was isolated in 18 specimens, and showed $100 \%$ resistance to penicillin and $44.4 \%$ (eight isolates) resistance to trimethoprim-sulphamethaxazole. Seven isolates (38.9\%), however, showed sensitivity to trimethoprim-sulphamethaxazole. There was $83.3 \%$ (15 isolates) with sensitivity to cloxacillin, while $11.1 \%$ (two isolates) showed resistance.

Clindamycin was effective in $77.8 \%$ (14 isolates) but resistance was noted in 16.7\% (3 isolates). Erythromycin/ azithromycin was also effective against staphylococci, showing sensitivity rates of $72.2 \%$ (13 isolates) and a

\begin{tabular}{|c|c|c|}
\hline Micro-organism & No of isolates & Percentages \\
\hline Acinetobacter baumannii complex & 2 & 1 \\
\hline Burkholderia cepacia & 3 & 1.4 \\
\hline Escherichia coli & 1 & 0.5 \\
\hline Klebsiella oxytoca & 1 & 0.5 \\
\hline Klebsiella pneumoniae & 3 & 1.4 \\
\hline Proteus species & 1 & 0.5 \\
\hline Pseudomonas aeruginosa & 3 & 1.4 \\
\hline Pseudomonas putida & 1 & 0.5 \\
\hline Coagulase negative staphylococcus & 9 & 4.3 \\
\hline Bacteriodes & 35 & 16.7 \\
\hline Enterobacter cloacae complex & 4 & 1.9 \\
\hline Gram negative bacillus & 6 & 2.9 \\
\hline Haemophilus parainfluenzae & 1 & 0.5 \\
\hline Morganella morganii & 8 & 3.8 \\
\hline Cornybacterium species & 4 & 1.9 \\
\hline Gemella morbillorum & 1 & 0.5 \\
\hline Micrococcus species & 1 & 0.5 \\
\hline Staphylococcus species & 4 & 1.9 \\
\hline Staphylococcus aureus & 18 & 8.6 \\
\hline Candida albicans & 3 & 1.4 \\
\hline Yeast - not Candida albicans & 3 & 1.4 \\
\hline Normal oral flora & 1 & 0.5 \\
\hline Staphylococcus epidermidis & 7 & 3.3 \\
\hline Staphylococcus haemolyticus & 3 & 1.4 \\
\hline Streptococcus anginosus & 8 & 3.8 \\
\hline Streptococcus alpha haemolytic & 7 & 3.3 \\
\hline Streptococcus beta haemolytic & 6 & 2.9 \\
\hline Streptococcus bovis & 4 & 1.9 \\
\hline Streptococcus constellatus & 10 & 4.8 \\
\hline Streptococcus cristatus & 1 & 0.5 \\
\hline Streptococcus group A & 3 & 1.4 \\
\hline Streptococcus group C & 1 & 0.5 \\
\hline Streptococcus group F & 4 & 1.9 \\
\hline Streptococcus mitis/oralis & 8 & 3.8 \\
\hline Streptococcus non-haemolytic & 6 & 2.9 \\
\hline Streptococcus pyogenes & 1 & 0.5 \\
\hline Streptococcus sanguinis & 1 & 0.5 \\
\hline Streptococcus warneri & 1 & 0.5 \\
\hline Viridans streptococcus & 25 & 11.9 \\
\hline
\end{tabular}

\section{Number of cases presenting with single} fascial space involvement

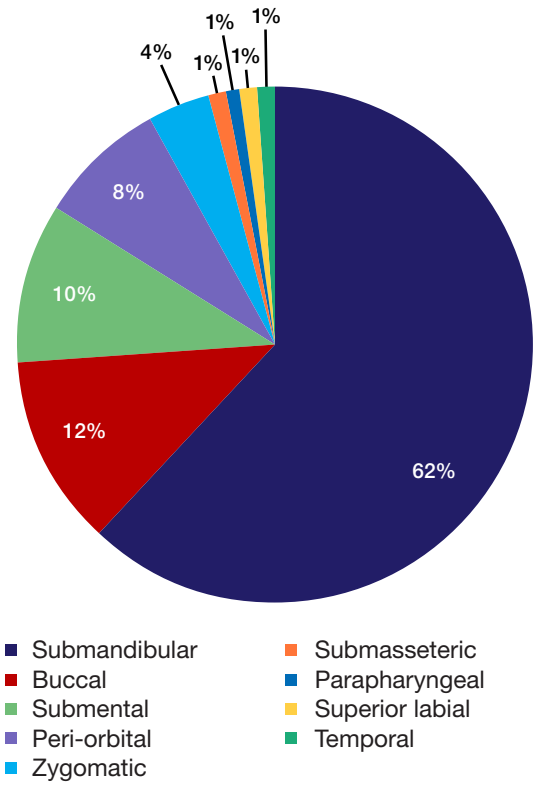

Figure 1. Number of cases presenting with single fascial space involvement.

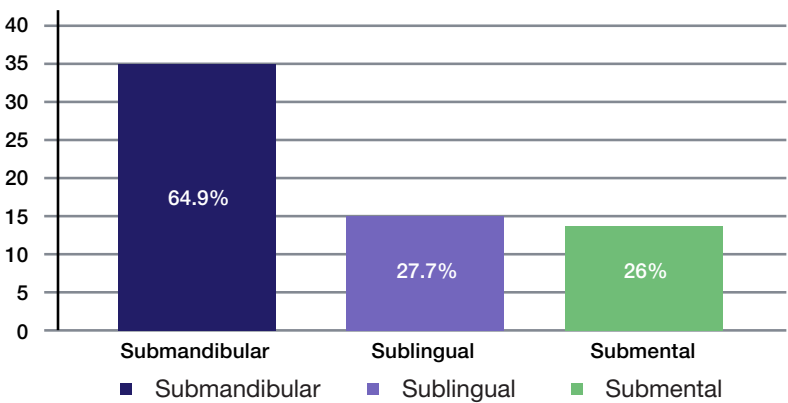

Figure 2. The most common fascial spaces implicated in multiple space involvement.

\begin{tabular}{|l|c|c|c|}
\hline \multicolumn{4}{|l|}{ Table 2. Antimicrobial sensitivity of Bacteroides species. } \\
\hline Antimicrobial & Tested & Sensitive & Resistant \\
\hline Amoxicillin-clavulanic acid & 35 & 35 & 0 \\
\hline Clindamycin & 35 & 35 & 0 \\
\hline Metronidazole & 35 & 35 & 0 \\
\hline Cefoxitin & 35 & 35 & 0 \\
\hline Chloramphenicol & 35 & 35 & 0 \\
\hline
\end{tabular}

Table 3. Antimicrobial sensitivity of Staphylococcus aureus.

\begin{tabular}{lccccc|} 
Antimicrobial & Tested & Sensitive & Resistant & $\begin{array}{c}\text { Intermediate } \\
\text { sensititve }\end{array}$ \\
\hline Penicillin & 18 & 0 & 18 & 0 \\
\hline Erythromycin & 18 & 13 & 3 & 2 \\
\hline Clindamycin & 18 & 14 & 3 & 1 \\
\hline Cloxacillin & 18 & 15 & 2 & 1 \\
\hline Vancomycin & 18 & 1 & 0 & 17 \\
\hline
\end{tabular}

Table 4. Antimicrobial sensitivity of Viridans streptococci.

\begin{tabular}{|c|c|c|c|c|c|}
\hline & Antimicrobial & Tested & Sensitive & Resistant & $\begin{array}{c}\text { Intermediate } \\
\text { sensititve }\end{array}$ \\
\hline \multirow[t]{3}{*}{$1^{\text {st }}$ Line } & Penicillin & 21 & 7 & 1 & 13 \\
\hline & Erythromycin & 21 & 15 & 6 & 0 \\
\hline & Clindamycin & 21 & 16 & 4 & 1 \\
\hline \multirow[t]{3}{*}{$2^{\text {nd }}$ Line } & Cefotaxime & 21 & 4 & 1 & 16 \\
\hline & Vancomycin & 21 & 4 & 0 & 17 \\
\hline & Linezolid & 21 & 1 & 0 & 20 \\
\hline
\end{tabular}


low resistance of $5.5 \%$ (two isolates). With regard to tetracycline, 3 isolates (16.7\%) were resistant and 38.9\% (seven isolates) were found to be sensitive Only one case $(5.5 \%)$ was sensitive to vancomycin.

Other commonly isolated microbes and their sensitivities patterns are displayed in Tables 2, 3 and 4 respectively.

\section{Sensitivity of isolates to commonly prescribed antibiotics}

Twenty two of the isolates were sensitive to amoxicillin with clavulanic acid (15.2\%), 28 were sensitive to penicillin (19.3\%) and 61 to erythromycin/azithromycin (42.1\%). The majority of the isolates (95) were sensitive to clindamycin (65.5\%). Results are shown in Figure 3.

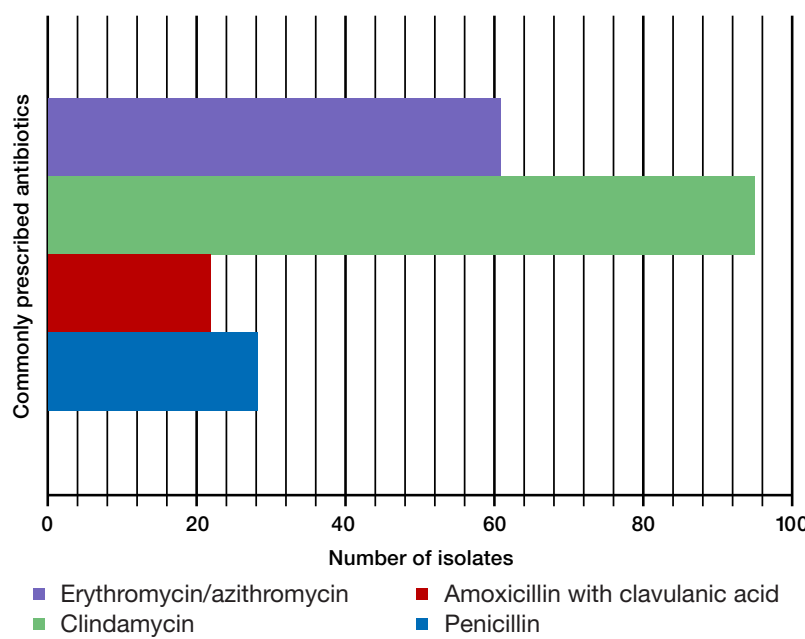

Figure 3. Sensitivity of commonly prescribed antibiotics.

\section{Statistical analysis}

Statistically significant association was observed between age and aetiology $(p=0.000)$; age and fascial space $(p=$ $0.005)$, gender and presence of microorganism $(p=0.007)$.

The presence of microorganisms was statistically significantly associated with antibiotic sensitivity $(p=0.000)$ and resistance $(p=0.000)$ profiles. Similarly the gender was significantly associated with the antibiotic sensitivity profiles.

\section{DISCUSSION}

Head and neck infections may result in serious morbidity and mortality. Early recognition of these infections, a thorough understanding of microorganisms involved and their sensitivity patterns are critical in their efficient management. In addition to surgical incision and drainage, antibiotic therapy is vital for successful treatment.

In order to administer antibiotics effectively, microbiological data on the infection is required. However, information on the microbiology and antibiotic susceptibility requires time, and subsequently antibiotics are administered prior to obtaining the aspirate results. ${ }^{11}$

Thus, the selection of the appropriate antibiotics is essential for successful treatment of these infections. The purpose of this study was to identify the microbial flora and antibiotic sensitivity patterns in patients with head and neck space infections and in so doing, provide a better understanding on the management of these infections.

In the current study, 145 patients who had pus aspirates and swabs taken were selected as the sample population. The age of the patients that commonly presented with head and neck space infections ranged from 21 to 40 years. This outcome is similar to the age distribution described in other published works. ${ }^{3,4,5}$ Similar to the findings of previous studies, $2,5,7,8$ head and neck space infections were more common in males than females in our study.

Our study showed single fascial spaces to be more commonly involved in the head and neck space infections than multiple spaces. This result corroborates the findings of $\mathrm{Ye}$ et al. ${ }^{11}$ Conversely, lbeyemi et al. ${ }^{12}$ reported multiple space involvement as being more common. In the current study, the most common single space involved was the submandibular space, followed by the buccal and submental space, a finding that is in agreement with that of Singh et al. ${ }^{8}$

A South African study by Molomo et al. ${ }^{5}$ showed the submandibular space to be the most commonly involved followed by the submental space. While Molomo et al. ${ }^{5}$ did not report on multiple fascial space involvement, our study showed that the submandibular, sublingual and submental spaces were most often implicated in multiple fascial space involvement. However the submandibular space was reported as the most common followed by the submental and lateral pharyngeal spaces by other researchers. ${ }^{4,6}$

Most infections in our study were non-odontogenic in origin (56\%). This is contrary to the findings by BoscoloRizzo et al. ${ }^{1}$ who reported odontogenic infection as the most common cause. Odontogenic causes of infection in the current study comprised $50 \%$ periapical lesions, $34 \%$ alveolar osteitis, $10 \%$ pericoronitis and $6 \%$ periodontitis. In contrast, a previous study reported $71 \%$, $17 \%, 6 \%$, and $1 \%$ of odontogenic causes of infection to be due to pulpitis, periodontitis, alveolar osteitis and needle tracts respectively. ${ }^{2}$

Bacteroides species was the most commonly isolated anaerobic bacterium in this study and was found in $15 \%$ of cases. Although not the most commonly isolated microorganism in other studies, Bacteroides fragilis and Bacteroides corrodens did appear in smaller concentrations of $5 \%$ and $2.5 \%$ of cases respectively, in the study by Walia et al. ${ }^{10}$ In contrast, the most commonly isolated microorganisms in a study by Molomo et al. ${ }^{5}$ were aerobic.

In the current study, Viridans streptococci were the second most isolated microorganisms, which contradicts other studies where Streptococcus viridans species were the most isolated microorganisms. ${ }^{5-7}$

Staphylococcus aureus was the third most isolated microorganism in our study in $8.6 \%$ cases. This finding is contrary to previous studies where Staphylococcus 
aureus was found in higher numbers. A study by Molomo et al. ${ }^{5}$ reported $14 \%$ cases while Shah et al. ${ }^{7}$ reported $16 \%$. Staphylococcus aureus observed in our study could have occurred secondary to contamination from the skin and introduced during treatment. The isolation of Staphylococcus aureus has clinical significance, as strains resistant to routinely used antibiotics have been reported..$^{13}$

Similarly to Molomo et al. ${ }^{5}$ the gram negative aerobes isolated in our study included Klebsiella, Enterobacter and Pseudomonas species. The presence of gram negative bacilli was also reported by Walia et al. ${ }^{10}$ In addition, Prevotella was the most commonly isolated gram negative bacillus in a study by Singh et al. ${ }^{8}$ occurring in $25.81 \%$ of the specimens.

One unanticipated finding was the identification of Morganella morganii complex which were isolated in 3\% of cases. Although Morganella morganii has a wide distribution, it is considered an uncommon cause of community-acquired infection and it is most often encountered in postoperative and other nosocomial infections such as urinary tract infections. This microorganism was isolated in an aspirate from an HIV positive patient.

The source of infection was a mandibular reconstruction plate that had caused chronic sepsis. A previous study by $\mathrm{Ho}$ et al. ${ }^{14}$ also reported a rare case of Ludwig's angina caused by Morganella Morgani. Enterobacter cloacae is a member of the normal gut flora in many humans and is not usually a primary pathogen.

Some strains have been associated with urinary and respiratory tract infections in immunocompromised individuals. ${ }^{15}$ Candida albicans was found in $1.4 \%$ of cases in our study, which is contrary to $5 \%$ and $2.5 \%$ occurrence in previous studies. 5,10

In the current study, Bacteroides species was 100\% sensitive to metronidazole, carbapenems, piperacillin, clindamycin, cefoxitin, chloramphenicol and amoxicillin with clavulanic acid. The South American study by Fernandez-Canigia et al. ${ }^{16}$ showed similar high sensitivity rates of $100 \%$ to metronidazole and tigecycline, 99\% to imipenem and piperacillin-tazobactam, 96\% to ampicillinsulbactam and $91 \%$ to moxifloxacin. However, lower sensitivity rates against cefoxitin (72\%) and clindamycin (52\%) were also observed.

Viridans streptococci displayed highest sensitivities to erythromycin/azithromycin (60\%) and clindamycin (64\%). In contrast, Shah et al. $^{7}$ reported high sensitivity levels of $100 \%$ to carbenicillin, amikacin and imipenem and $89.4 \%$ to ceftriaxone. Our results also differed from the findings of Rega et al. ${ }^{6}$ and Molomo et al. ${ }^{5}$ where the highest sensitivity rates to penicillin was reported as $87.1 \%$ and $97 \%$ respectively.

In the current study, Staphylococcus aureus showed sensitivities of $83.3 \%$ to cloxacillin, $77.8 \%$ to clindamycin and $72.2 \%$ to erythromycin/azithromycin while Molomo et al. ${ }^{5}$ reported sensitivities of $70 \%, 90 \%$ and $83 \%$ respectively. Although Molomo et al. ${ }^{5}$ reported $31 \%$ resistance, this microbe showed $100 \%$ resistance to penicillin.
Shah et al. ${ }^{7}$ reported a higher sensitivity rate of $100 \%$ to amoxicillin with clavulanic acid and a lower resistance of $31.3 \%$ to amoxicillin.

Clindamycin was found to be highly effective against the three most commonly isolated bacteria in the current study. This efficacy was reiterated in studies by Bahl et al. ${ }^{3}$, Fating et $a l .{ }^{4}$, Molomo et $a l .{ }^{5}$ and Singh et al. ${ }^{8}$ in which aerobic organisms isolated were sensitive to clindamycin. However, severe side effects like pseudomembranous colitis and the emergence of antibiotic resistance, make this antibiotic unsuitable as the first line drug for the treatment of head and neck space infections. Clindamycin should therefore be reserved for severe penicillin resistant infections to curtail the increasing resistance.

Viridans streptococci and Staphylococcus aureus showed high sensitivity rates to erythromycin/azithromycin in agreement with the findings reported by Molomo et al. ${ }^{5}$ However the opposite result was obtained in the study by Singh et al. ${ }^{8}$ where all isolates displayed low sensitivity rates to erythromycin (38.89\%).

There was a statistically significant association between age and aetiology $(p=0.000)$. Older patients in the 51-60 and greater than 60 years age groups presented more commonly with unknown causes of infection. The younger patients in the 0-20 and 21-30 years age groups were more commonly affected by odontogenic infections including alveolar osteitis, periodontal and periapical abscesses.

This finding could be ascribed to the low socio-economic groups comprising the majority of patients presenting at public hospitals. These patients are inadequately exposed to oral health education and are at greater risk for the development of tooth decay and the associated odontogenic infections. This finding was reiterated in the study by Bahl et al. ${ }^{3}$, who reported the highest incidence of odontogenic infections in patients in the third decade of life.

There was likewise a statistically significant association between the age and fascial space $(p=0.005)$, with the majority of patients presenting with single space involvement. This correlates with the findings of Walia et al. ${ }^{10}$, where single space involvement was significantly more common. Patients younger than 40 years presented more commonly with single space involvement, while the older patients in the $5^{\text {th }}$ decade of life presented more commonly with multiple space involvement.

The association between gender and the presence of microorganisms was also statistically significant $(p=$ 0.007). The majority of specimens were acquired from males. This was consistent with studies by Bahl et al. ${ }^{3}$, Fating et al. ${ }^{4}$ and Walia et al. ${ }^{10}$ where the majority of isolates were obtained from male patients. However, more microorganisms were identified in females (45 of 48 patients or $94 \%$ ) than in males (72 of 97 patients or $74 \%$ ).

Moreover there was statistically significant association between the presence of microorganisms and antibiotic sensitivity $(p=0.000)$ and resistance $(p=0.000)$ profiles. 
This is understandable, as the microorganisms isolated would have displayed either sensitivity or resistance to a specific antibiotic.

It was similarly noted that gender of the patient was significantly associated with antibiotic sensitivity profiles. Thirty nine of 48 female patients (81.2\%) that presented with head and neck space infections displayed microorganisms that were sensitive to particular antibiotics.

\section{CONCLUSION}

The predominant microorganisms responsible for head and neck infections were gram positive facultative anaerobes. Bacteroides species were the most commonly isolated bacteria, followed by Viridans streptococci and Staphylococcus aureus.

Most infections occurred in the third and fourth decades of life. Patients older than 50 years presented more commonly with non-odontogenic causes of infection, while younger patients more frequently presented with odontogenic causes of infection. Patients younger than 40 years presented more commonly with single space involvement, while the older patients in the $5^{\text {th }}$ decade of life presented more commonly with multiple space involvement. Microorganisms were more frequently isolated in females than males and in patients in the age range of 21-40 years.

The most commonly isolated microorganisms (Bacteroides species, Staphylococcus aureus, Viridans streptococci) in the current study were sensitive to clindamycin. Bacteroides species was found to be $100 \%$ sensitive to amoxicillin with clavulanic acid.

\section{Limitations}

This was a retrospective study which limited the variables that could be studied. The method of collection of the aspirates may have influenced the microbiology results, especially if the anatomical site of collection was not adequately cleaned with an alcohol swab prior to the procedure.

Since the Maxillofacial clinic at Livingstone Hospital is a tertiary care centre, it is likely that most patients included in this study would have received antibiotics prior to admission. Therefore the emergence of resistant strains due to administration of these antimicrobial agents may not have been accurately assessed.

\section{Recommendations}

A longer study period with a larger sample size evaluating the microbial spectrum and resistance patterns may be necessary to monitor developing trends.

Since odontogenic infection was found to be a common cause of head and neck space infections, particularly in the third and fourth decades of life, oral health education should further emphasise the importance of caries prevention, good oral hygiene practices, early presentation and intervention to minimise the complications of pulpitis.

\section{References}

1. Boscolo-Rizzo P, Da Mosto MC. Submandibular space infection: a potentially lethal infection. Inter J Infec Dis. 2009; 13: 327-33.

2. Mathew GC, Ranganathan LK, Ghandi S et al. Odontogenic maxillofacial space infections at a tertiary care centre in North India: a five year retrospective study. Int $J$ Infec Dis. 2012; 16: e296-e302.

3. Bahl R, Sandhu S, Singh K, Sahai N, Gupta M. Odontogenic infections: Microbiology and management. Contemp Clin Dent. 2014; 5: 307-11.

4. Fating NS, Saikrishna D, Vijay Kumar GS, Shetty SK, Rao MR. Detection of bacterial flora in orofacial space infections and their antibiotic sensitivity profile. J Maxillofac Oral Surg. 2014; 13: $525-32$.

5. Molomo EM, Motloba DP, Bouckaert MM, Tlholoe MM. Bacteriology and management of orofacial infections in a Maxillofacial and Oral Surgery Clinic, South Africa. S Afr Dent J. 2016; 71: 474-7.

6. Rega AJ, Aziz SR, Ziccardi VB. Microbiology and antibiotic sensitivities of deep neck space infections. J Oral Maxillofac Surg. 2004; 62: 25-6.

7. Shah A, Ramola V, Nautiyal V. Aerobic microbiology and culture sensitivity of head and neck space infection of odontogenic origin. Nat J Maxillofac Surg. 2016; 7: 56-61.

8. Singh M, Kambalimath DH, Gupta KC. Management of odontogenic space infection with microbiology study. J Maxillofac Oral Surg. 2014; 13: 133-9.

9. Cabral M, Gowrishankar S, Amerally P. Investigation of the microbiology and antibiotic sensitivity of skin and soft tissue infections of the head and neck region. $\mathrm{Br}$ Oral Maxillofac Surg. 2016; 54: 124-5.

10. Walia IS, Borle RM, Mehendiratta D, Yadav AO. Microbiology and antibiotic sensitivity of head and neck space infections of odontogenic origin. J Maxillofac Oral Surg. 2013; 13: 16-21.

11. Ye L, Liu Y, Geng A-L, Fu H-Y. Microbiological examination to investigate the differences in microorganisms and anti biotic sensitivity of head and neck space infections. Biomed Res. 2017; 28: 290-4.

12. Ibeyemi ST, Okoje-Adesomoju VN, Dada-Adegbola $\mathrm{HO}$, Arotiba JT. Pattern of orofacial bacterial infections in a tertiary hospital in southwest Nigeria. J West Afr Coll Surg. 2014; 4: 112-41.

13. Costa SF, Miceli MH, Anaissie EJ. Mucosa or skin as source of coagulase-negative Staphylococcal bacteraemia? Lancet Infec Dis. 2004; 4: $278-86$.

14. Ho MP, Tsai KC, Yen SL, Lu CL, Chen CH. A rare cause of Ludwig's angina by Morganella morganii. J Infec. 2006; 53: e191-4.

15. Stock I, Gruger T, Weidemann, B. Natural antibiotic susceptibility of strains of the Enterobacter cloacae complex. Int $\mathrm{J}$ Antimicr Agents. 2001; 18: 537-45.

16. Fernadez-Canigia L, Legaria MC, Castello $L$ et al. First national survey of antibiotic susceptibility of the Bacteroides fragilis group: Emerging resistance to carbapenems in Argentina. Antimicr Agents Chem. 2012; 56: 1309-14. 